\title{
Pro-life role for c-Jun N-terminal kinase and p38 mitogen-activated protein kinase at rostral ventrolateral medulla in experimental brain stem death
}

Alice YW Chang

\begin{abstract}
Background: Based on an experimental brain stem death model, we demonstrated previously that activation of the mitogen-activated protein kinase kinase 1/2 (MEK1/2)/extracellular signal-regulated kinase 1/2 (ERK1/2)/ mitogen-activated protein kinase signal-interacting kinase 1/2 (MNK1/2) cascade plays a pro-life role in the rostral ventrolateral medulla (RVLM), the origin of a life-and-death signal detected from systemic arterial pressure, which sequentially increases (pro-life) and decreases (pro-death) to reflect progressive dysfunction of central cardiovascular regulation during the advancement towards brain stem death in critically ill patients. The present study assessed the hypothesis that, in addition to ERK1/2, c-Jun NH2-terminal kinase (JNK) and p38 mitogen-activated protein kinase (p38MAPK), the other two mammalian members of MAPKs that are originally identified as stress-activated protein kinases, are activated specifically by MAPK kinase 4 (MAP2K4) or MAP2K6 and play a pro-life role in RVLM during experimental brain stem death. We further delineated the participation of phosphorylating activating transcriptional factor-2 (ATF-2) and c-Jun, the classical transcription factor activated by JNK or P38MAPK, in this process.
\end{abstract}

Results: An experimental model of brain stem death that employed microinjection of the organophosphate insecticide mevinphos (Mev; $10 \mathrm{nmol}$ ) bilaterally into RVLM of Sprague-Dawley rats was used, alongside cardiovascular, pharmacological and biochemical evaluations. Results from ELISA showed that whereas the total JNK, p38MAPK, MAP2K4 and MAP2K6 were not affected, augmented phosphorylation of JNK at Thr183 and Tyr185 and p38MAPK at Thr180 and Tyr182, accompanied by phosphorylation of their upstream activators MAP2K4 at Ser257 and Thr261 and MAP2K6 at Ser207 and Thr211 in RVLM occurred preferentially during the pro-life phase of experimental brain stem death. Moreover, the activity of transcription factors ATF-2 at Thr71 and c-Jun at Ser73, rather than Elk-1 at Ser383 in RVLM were also augmented during the pro-life phase. Furthermore, pretreatment by microinjection into the bilateral RVLM of specific JNK inhibitors, JNK inhibitor I (100 pmol) or SP600125 (5 pmol), or specific p38MAPK inhibitors, p38MAPK inhibitor III (500 pmol) or SB203580 (2 nmol), exacerbated the depressor effect and blunted the augmented life-and-death signal exhibited during the pro-life phase. On the other hand, pretreatment with the negative control for JNK or p38MAPK inhibitor, JNK inhibitor I negative control (100 pmol) or SB202474 (2 nmol), was ineffective in the vehicle-controls and Mev-treatment groups.

Conclusions: Our results demonstrated that activation of JNK or p38MAPK in RVLM by their upstream activators MAP2K4 or MAP2K6 plays a preferential pro-life role by sustaining the central cardiovascular regulatory machinery during experimental brain stem death via phosphorylation and activation of nuclear transcription factor ATF-2 or c-Jun.

\footnotetext{
* Correspondence: cgmf.kmc@gmail.com

Center for Translational Research in Biomedical Sciences, Kaohsiung Chang

Gung Memorial Hospital, Kaohsiung 83301, Taiwan, Republic of China
} 


\section{Background}

Whereas brain stem death is the legal definition of death in the United States of American [1], United Kingdom [2], European [3], Taiwan and many other countries $[1,4]$, the detailed cellular and molecular mechanisms underlying this phenomenon of prime medical importance are only begun to emerge. Since asystole invariably occurs within hours or days after the diagnosis of brain stem death [5], it is strongly suggested that permanent impairment of the brain stem cardiovascular regulatory machinery precedes death [6]. Further understanding of the mechanisms of this aspect of cardiovascular regulatory dysfunction should therefore enrich the dearth of information currently available on brain stem death.

Mitogen-activated protein kinases (MAPKs) are serine/threonine-specific protein kinases that regulate proliferation, gene expression, differentiation, cell survival and apoptosis [7]. Three most widely characterized MAPK subfamilies are extracellular signal-regulated kinase 1/2 (ERK1/2), c-Jun NH2-terminal kinase (JNK) and p38MAPK [8]. Activation of MAPKs requires phosphorylation of its regulatory loop by upstream activators. Thus, each of these subfamilies is composed of MAPK kinase kinase (MAP3K) that, on activation, phosphorylates a MAPK kinase (MAP2K), then a MAPK. The phosphorylated MAPK interacts with its cellular substrates, which translocate to the nucleus to modulate transcription factors that results in a diverse range of biological responses.

Based on a clinically relevant animal model of brain stem death $[6,9]$ in conjunction with toxicity elicited by the organophosphate insecticide mevinphos (3-(dimethoxyphosphinyloxyl)-2-butenoic acid methyl ester (Mev), a US Environmental Protection Agency Toxicity Category I pesticide, we demonstrated previously that the rostral ventrolateral medulla (RVLM) is a suitable neural substrate for mechanistic evaluation of this fatal phenomenon [6], because it is the origin of a life-and-death signal [10] that reflects failure of the central cardiovascular regulatory machinery during brain stem death [11-13] and is a brain stem site via which Mev acts to elicit cardiovascular toxicity [9]. Of interest is that the waxing and waning of the life-and-death signal, which mirrors the fluctuation of neuronal functionality in RVLM, presents itself as the low-frequency (LF) component in the systemic arterial pressure (SAP) spectrum of comatose patients [11-13]. More importantly, the distinct phases of augmentation followed by reduction of the LF power exhibited during Mev intoxication [14-17] can be designated the pro-life and pro-death phase of central cardiovascular regulation in this model of brain stem death [6]. Based on this model, our laboratory has previously demonstrated that activation of MAPK kinase 1/2 (MEK1/2) in RVLM, followed by ERK1/2 and MAPK signal-interacting kinase $1 / 2$ (MNK1/2) activation, is responsible for the pro-life phase by sustaining the central cardiovascular regulatory machinery during brain stem death $[18,19]$.

Of the three MAPKs characterized in mammals, JNK and p38MAPK are originally identified as a stressactivated protein kinase (SAPK) that primarily mediates inflammatory response $[20,21]$ and promotes cell death [22-24]. However, recent studies further suggest that JNK and p38MAPK may also participate in cell survival [25-27], proliferation [28] or pressor response [29]. With particular relevance to the present study, simultaneous inhibition of JNK and p38MAPK increases cell death in the heart of rats induced by ischemia/reperfusion injury [30]. Moreover, activation of p38MAPK signaling pathway in RVLM underlies the pressor response to angiotensin II (Ang II) in rats [29].

As death represents the end of existence for an individual, we proposed previously [6] that multiple pro-life and pro-death programs must be activated in RVLM during the progression toward brain stem death. Furthermore, we previously demonstrated that ERK $1 / 2$ in RVLM plays a pro-life role in experimental brain stem death $[18,19]$. In our continual search for the cellular and molecular underpinning of brain stem death, the next logical direction is to evaluate the contribution of the other two family members of MAPKs, JNK or p38MAPK in RVLM to this fatal phenomenon.

Based on our Mev intoxication model [6], the present study evaluated the hypothesis that JNK and p38MAPK in RVLM play a pro-life role during brain stem death. We further delineated the upstream participation of MAPK kinase 4 (MAP2K4) and MAPK kinase 6 (MAP2K6) and downstream participation of transcription factors activating transcriptional factor-2 (ATF-2) and c-Jun, the nuclear substrates of JNK or p38MAPK [31] in this process. Our results demonstrated that activation of JNK and p38MAPK in RVLM plays a preferential pro-life role by sustaining central cardiovascular regulatory functions during brain stem death. We further found that the signaling cascade for the pro-life process includes upstream phosphorylation of MAP2K4 or MAP2K6, and downstream activation of transcription factors ATF-2 or c-Jun.

\section{Methods}

Adult male Sprague-Dawley rats $(275-350$ g, $\mathrm{n}=129)$ purchased from the Experimental Animal Center of the National Science Council, Taiwan, Republic of China were used. They were housed in our Association for Assessment and Accreditation of Laboratory Animal Care (AAALAC)-International accredited Center for Laboratory Animals. All animal care and experimental procedures carried out in this study have been approved by 
the Institutional Animal Care and Use Committee of the Kaohsiung Chang Gung Memorial Hospital, and were in compliance with the guidelines of this Committee. Animals were housed in groups of two to three in individually ventilated cages, in a temperature-controlled room $\left(22 \pm 2^{\circ} \mathrm{C}\right.$ ) with $12 \mathrm{~h}$ light $/ 12 \mathrm{~h}$ dark cycles (lights on at 07:00 h), with free access to rat chow and water. All efforts were made to minimize animal suffering and to reduce the number of animal used.

\section{General preparation}

After application of an induction dose of pentobarbital sodium (50 mg/kg, i.p.), preparatory surgery, including cannulation of a femoral artery and a femoral vein, together with tracheal intubation, was carried out. During the recording session, which routinely commenced 60 min after the administration of pentobarbital sodium, anesthesia was maintained by intravenous infusion of propofol (Zeneca, Macclesfield, UK) at $20-25 \mathrm{mg} / \mathrm{kg} / \mathrm{h}$. We have demonstrated previously [32] that this scheme provided satisfactory anesthetic maintenance while preserving the capacity of central cardiovascular regulation. Rats were allowed to breathe spontaneously with room air and body temperature of rats was maintained at $37^{\circ} \mathrm{C}$ with a heating pad.

\section{Animal model of brain stem death}

The Mev intoxication model of brain stem death [6] that we established previously was used. Since Mev induces comparable cardiovascular responses on given systemically or directly to RVLM [9], we routinely microinjected Mev bilaterally into RVLM to elicit site-specific effects [9,14-17]. SAP signals recorded from the femoral artery were simultaneously subject to on-line power spectral analysis (SPA10a; Notocord, Croissy-Sur-Seine, France) $[9,14-17,33]$. We were particularly interested in the LF component $(0.25-0.8 \mathrm{~Hz})$ in the SAP spectrum because its power density mirrors the prevalence of baroreflexmediated sympathetic neurogenic vasomotor discharges that emanate from this brain stem site [33]. More importantly, our laboratory demonstrated previously [14-17] that the power density of this spectral signal exhibits biphasic changes that reflect the pro-life and pro-death phases seen during the progression towards brain stem death in patients who succumbed to organophosphate poisoning [13]. Heart rate (HR) was derived instantaneously from SAP signals. Temporal changes in the power density of the LF component, pulsatile SAP, mean SAP (MSAP) and HR were routinely followed for $180 \mathrm{~min}$ after Mev administration in an on-line and real-time manner.

\section{Microinjection of test agents}

Microinjection bilaterally of test agents into RVLM, each at a volume of $50 \mathrm{nl}$, was carried out stereotaxically and sequentially [9,14-17] via a glass micropipette connected to a $0.5-\mu$ l Hamilton (Reno, NV, USA) microsyringe. The coordinates used were: $4.5-5 \mathrm{~mm}$ posterior to lambda, $1.8-2.1 \mathrm{~mm}$ lateral to midline, and $8.1-8.4 \mathrm{~mm}$ below the dorsal surface of cerebellum. These coordinates were selected to cover the ventrolateral medulla at which functionally identified sympathetic premotor neurons reside [34]. Test agents used included Mev (kindly provided by Huikwang Corporation, Tainan, Taiwan), two specific JNK inhibitors, JNK inhibitor I (Calbiochem, San Diego, CA, USA) [35] and JNK inhibitor II (SP600125, Calbiochem) [36]; two specific p38MAPK inhibitors, p38 MAPK inhibitor III (Calbiochem) [37] and SB203580 (Calbiochem) [38]; and negative controls, JNK inhibitor I negative control (Calbiochem) [35] or SB202474 (Calbiochem) [38]. All test agents used for pretreatment were given 30 min before the administration of Mev. The doses were adopted from previous reports [35-38] that used those test agents for the same purpose as in this study. Application of the same amount of artificial cerebrospinal fluid (aCSF) controlled for possible volume or solvent effect. The composition of aCSF was $(\mathrm{mmol} / \mathrm{L}): \mathrm{NaCl} 117, \mathrm{NaHCO}_{3} 25$, glucose 11, $\mathrm{KCl} 4.7, \mathrm{CaCl}_{2} 2.5, \mathrm{MgCl}_{2} 1.2$ and $\mathrm{NaH}_{2} \mathrm{PO}_{4}$ 1.2. To avoid the confounding effects of drug interactions, each animal was subject routinely to only one pharmacological treatment scheme.

\section{Collection of tissue samples from ventrolateral medulla}

As in previous studies [14-17], we routinely collected tissue samples for subsequent biochemical evaluations during the peak of the pro-life phase and pro-death phase (Mev group), or 30 or $180 \mathrm{~min}$ after microinjection of aCSF into RVLM (vehicle control group). Animals were killed with an overdose of pentobarbital sodium and tissues from both sides of the ventrolateral medulla, at the level of RVLM (0.5-1.5 mm rostral to the obex), were collected by micropunches made with a $1 \mathrm{~mm}$ (i.d.) stainless-steel bore to cover the anatomical boundaries of RVLM. Medullary tissues collected from anesthetized animals without any treatment served as the sham-controls. The concentration of total proteins extracted from tissue samples was determined by the BCA protein assay (Pierce, Rockford, IL, USA).

\section{ELISA for protein level of JNK, p38MAPK, MAP2K4, MAP2K6 or their phosphorylated forms}

Cell lysate from ventrolateral medulla was subject to a commercial kit for enzyme-linked immunosorbent assay (ELISA) according to the manufacturer's protocol to detect the levels of JNK1/2/3 (eBioscience, San Diego, CA, USA), phosphorylated JNK1/2/3 at Thr183/Tyr185 (eBioscience), p38MAPK (eBioscience), phosphorylated p38MAPK at Thr180/Tyr182 (eBioscience), MAP2K4 
(Antibodies-online, Atlanta, GA, USA), phosphorylated MAP2K4 at Ser257/Thr261 (TGR BioSciences, Thebarton, Australia), MAP2K6 (MyBioSource, San Diego, CA, USA) or phosphorylated MAP2K6 at Ser207/ Thr211 ( $\mathrm{R}$ and D Systems, Minneapolis, MN, USA). The final absorbance of reaction solution at $450 \mathrm{~nm}$ was determined by spectrophotometry using an ELISA microtiter plate reader (Anthros Labtec, Salzburg, Austria), and was expressed as fold changes against baseline-controls.

\section{Nuclear extract from ventrolateral medulla}

In some experiments, proteins from the nuclear fraction of the medullary samples were extracted using a commercial kit (Active Motif, Carlsbad, CA, USA). The concentration of protein in the nuclear extracts was again estimated by the BCA Protein Assay (Pierce).

\section{ELISA for activity of transcription factors ATF-2, c-Jun or Elk-1}

Nuclear extract from ventrolateral medulla was subject to a sensitive and specific commercial kit (Active Motif) for ELISA according to the manufacturer's protocol to detect the levels of phosphorylated c-Jun at Ser73, phosphorylated E twenty-six-like transcription factor 1 (Elk-1) at Ser383 or phosphorylated ATF-2 at Thr71. The final absorbance of the reaction solution at $450 \mathrm{~nm}$ was determined by spectrophotometry using an ELISA microtiter plate reader (Anthros Labtec), and expressed as fold changes against baseline-controls.

\section{Histology}

In some animals that were not used for biochemical analysis, the brain stem was removed at the end of the physiological experiment and fixed in 30\% sucrose in $10 \%$ formaldehyde-saline solution for at least $72 \mathrm{~h}$. Frozen $25-\mu \mathrm{m}$ sections of the medulla oblongata stained with neural red were used for histological verification of the microinjection sites.

\section{Statistical analysis}

All values are expressed as mean \pm SEM. The averaged value of MSAP or HR calculated every $20 \mathrm{~min}$ after the administration of test agents or aCSF, the sum total of power density for the LF component in the SAP spectrum over $20 \mathrm{~min}$, or the level or activity of protein or transcriptional factor in RVLM during each phase of experimental brain stem death, were used for statistical analysis. One-way or two-way ANOVA with repeated measures was used, as appropriate, to assess group means. This was followed by the Scheffé multiple-range test for post hoc assessment of individual means. $P<0.05$ was considered to be statistically significant.

\section{Results}

Mev intoxication model of brain stem death

We demonstrated previously that co-microinjection bilaterally of Mev (10 nmol) and aCSF into RVLM elicited a progressive depressor effect that became significant 100 min after application, accompanied by indiscernible alterations in HR. Concurrent changes in the power density of the LF component of SAP signals revealed two distinct phases [14-17]. The pro-life Phase I (MI) entailed a significantly augmented LF power that endured 80-100 min to reflect sustained brain stem cardiovascular regulatory functions. The pro-death Phase II (MII), which lasted the remainder of our 180-min observation period, exhibited further and significant reduction in the power density of this spectral component to below baseline, which signifies failure of central cardiovascular regulation that precedes brain stem death [6].

\section{Preferential activation of JNK in RVLM during the pro-life phase}

We first evaluated the fundamental premise that JNK in RVLM is activated during experimental brain stem death. Quantification by ELISA revealed that total JNK and its upstream activator MAP2K4 in ventrolateral medulla were not affected by microinjection of Mev into the bilateral RVLM (Figure 1). Interestingly, phosphorylated JNK (p-JNK) at Thr183 and Tyr185 in RVLM was significantly and preferentially augmented (Figure 1) during the pro-life phase of experimental brain stem death, which returned to baseline during the pro-death phase. However, phosphorylated MAP2K4 (p-MAP2K4) at Ser257/Thr261 was significantly increased (Figure 1) during both the pro-life and pro-death phases. The levels of JNK, MAP2K4 and phosphorylated JNK or MAP2K4 in ventrolateral medulla of vehicle groups $30 \mathrm{~min}$ (AI) or $180 \mathrm{~min}$ (AII) after aCSF application were comparable to sham-controls.

\section{Preferential activation of p38MAPK in RVLM during the pro-life phase}

We further evaluated whether p38MAPK in RVLM is also activated during experimental brain stem death. Quantification by ELISA again revealed that total p38MAPK and its upstream activator MAP2K6 in ventrolateral medulla were not affected by microinjection of Mev into the bilateral RVLM (Figure 2). Furthermore, both phosphorylated p38MAPK (p-p38MAPK) at Thr180/Tyr182 and phosphorylated MAP2K6 at Ser207/ Thr211 in RVLM were significantly augmented (Figure 2) during both pro-life and pro-death phase. The levels of p38MAPK, MAP2K6 and phosphorylated p38MAPK or MAP2K6 in ventrolateral medulla of vehicle groups after aCSF application were comparable to sham-controls. 

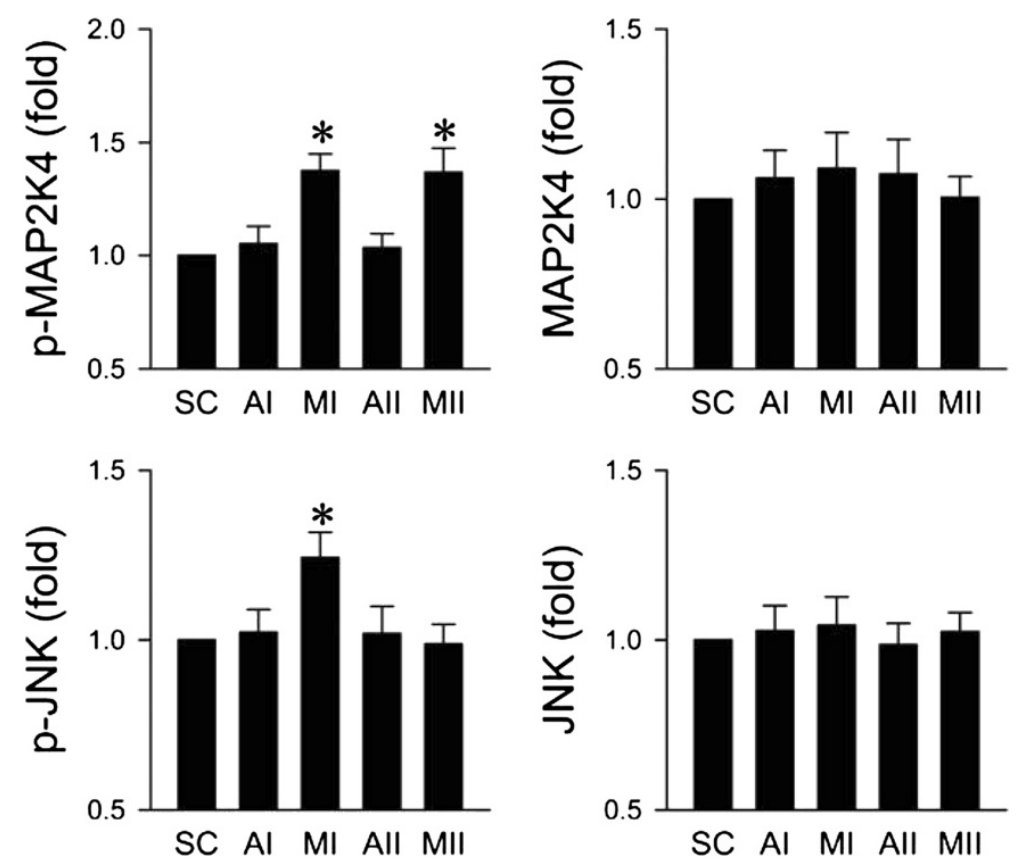

Figure 1 Activation of JNK and MAP2K4 in RVLM during the pro-life phase of experimental brain stem death. Changes in levels of total or phosphorylated JNK at Thr183 and Tyr185 and changes in levels of total or phosphorylated MAP2K4 at Ser257/Thr261 in folds relative to shamcontrol (SC), detected in ventrolateral medulla during the pro-life Phase I (MI) or pro-death Phase II (MII) during experimental brain stem death or during comparable time points after treatment with aCSF (AI or All). Values are presented as mean \pm SEM of triplicate analyses on tissue samples pooled from 5-7 animals in each experimental group. ${ }^{*} P<0.05$ versus corresponding aCSF group in the post hoc Scheffé multiple-range analysis.
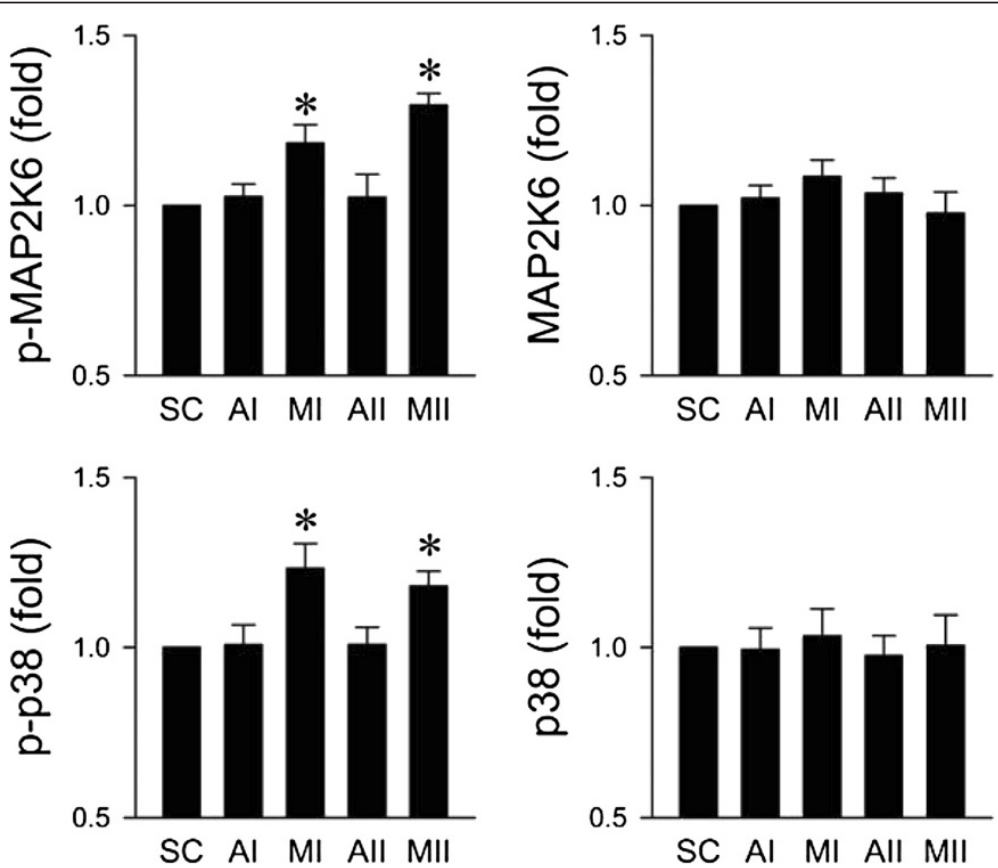

Figure 2 Activation of p38MAPK and MAP2K6 in RVLM during the pro-life phase of experimental brain stem death. Changes in levels of total or phosphorylated p38MAPK at Thr180 and Tyr182 and changes in levels of total or phosphorylated MAP2K6 at Ser207/Thr211 in folds relative to sham-control (SC), detected in ventrolateral medulla during the pro-life (MI) or pro-death (MII) phase of experimental brain stem death or during comparable time points in aCSF-controls (Al or All). Values are presented as mean \pm SEM of triplicate analyses on tissue samples pooled from 5-7 animals in each experimental group. ${ }^{*} P<0.05$ versus corresponding aCSF group in the post hoc Scheffé multiple-range analysis. 

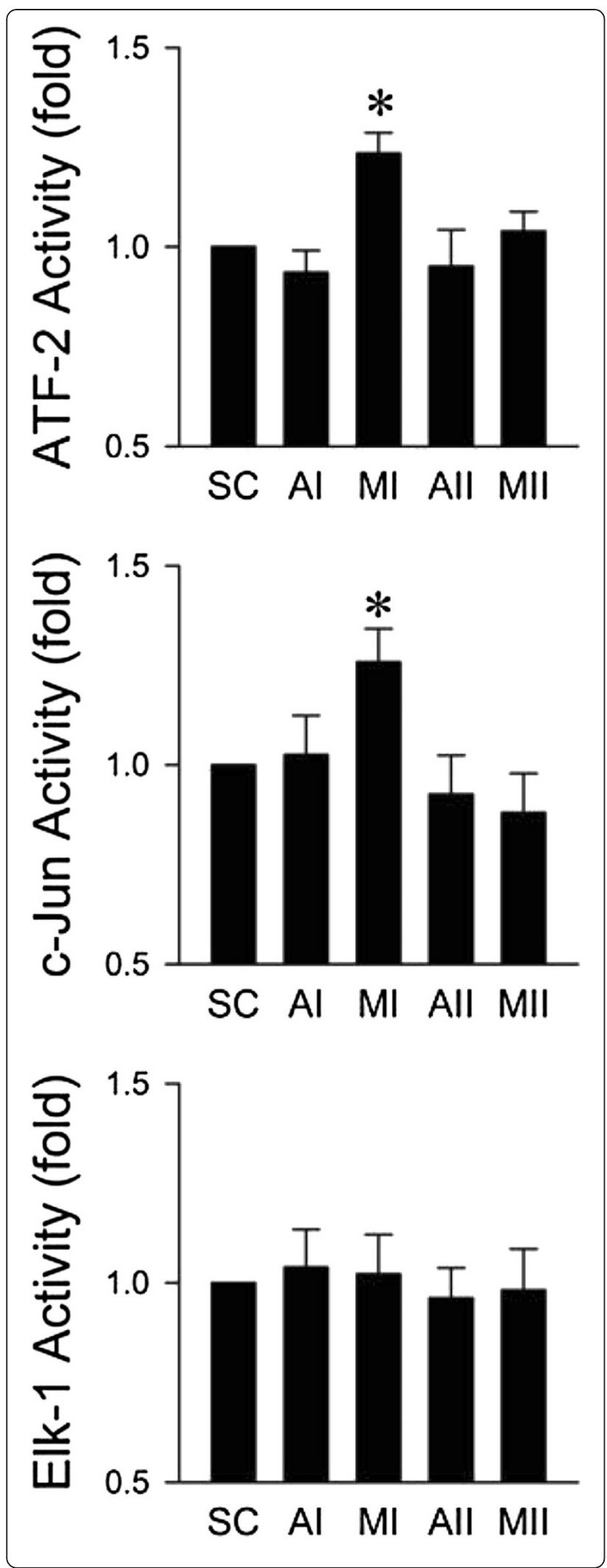

Figure 3 Activation of transcription factor ATF-2, c-Jun, rather than Elk-1 in RVLM during the pro-life phase of experimental brain stem death. Changes in the activity of ATF-2, c-Jun or Elk-1 represented by phosphorylation respectively at Thr71, Ser73 or Ser383, in folds relative to sham-control (SC), detected in ventrolateral medulla during the pro-life (MI) or pro-death (MII) phase of experimental brain stem death or during comparable time points in aCSF-controls (Al or All). Values are presented as mean \pm SEM of triplicate analyses on tissue samples pooled from 5-7 animals in each experimental group. ${ }^{*} P<0.05$ versus corresponding aCSF group in the post hoc Scheffé multiple-range analysis.

Preferential activation of transcription factors c-Jun, ATF-2, rather than Elk-1 in RVLM during the pro-life phase

We next determined the activity of transcription factors c-Jun, ATF-2 and Elk-1 in RVLM, which are activated by phosphorylated JNK or p38MAPK [39-41], during experimental brain stem death. Results from ELISA showed that significantly increased ATF-2 activity via phosphorylation at Thr71 in ventrolateral medulla was observed only during the pro-life phase (Figure 3). Similar results were obtained for augmented c-Jun activity via phosphorylation at Ser73, but not for Elk-1 activity as indicated by insignificant phosphorylation at Ser383. On the other hand, the activity of ATF-2, c-Jun or Elk-1 in ventrolateral medulla of aCSF-treatment group was comparable to sham-controls.

Activation of JNK in RVLM sustains central cardiovascular regulation during experimental brain stem death

Based on the stipulation that the magnitude and duration of the LF component of SAP signals during experimental brain stem death reflect the prevalence of the life-and-death signal [6], we next employed pharmacological blockade to evaluate whether a causal relationship exists between activation of JNK in RVLM and central cardiovascular regulation during brain stem death. Pretreatment with microinjection into the bilateral RVLM of JNK inhibitor I (100 pmol), a cell-permeable biological active peptide that binds specifically to JNK to inhibit phosphorylation of the activation domain of JNK and to prevent the activation of the downstream transcription factor c-Jun [35], exacerbated significantly the depressor effect and blunted the augmented power density of the LF component of SAP signals during the pro-life phase (Figure 4), without affecting HR. Similar results were obtained on local application bilaterally into RVLM of SP600125 (5 pmol), a cell-permeable, selective and reversible inhibitor of JNK [36] (Figure 4). Those pretreatments also significantly shortened the pro-life phase to 35$40 \mathrm{~min}$ by shifting the prevailing phase of the 180 -min observation period toward the pro-death phase (Figure 4). On the other hand, microinjection of JNK inhibitor I negative control (100 pmol) [35] into the bilateral RVLM did not significantly affect the increase in LF power during 
the pro-life phase nor the depressor effect and decrease in LF power already exhibited during the pro-death phase. Furthermore, pretreatments with aCSF or JNK inhibitor I negative control exerted no significant effects on the minimal cardiovascular responses in the aCSF-control group.

\section{Activation of p38MAPK in RVLM also sustains central cardiovascular regulation during experimental brain stem death}

We further applied the same experimental scheme to evaluate whether a causal relationship similarly exists between activation of p38MAPK in RVLM and central cardiovascular regulation during experimental brain stem death. Pretreatment with microinjection into the bilateral RVLM of p38MAPK inhibitor III (500 pmol), a potent, selective and ATP competitive p38MAPK inhibitor [37], also exacerbated significantly the depressor effect and blunted the augmented power density of the LF component of SAP signals during the pro-life phase (Figure 5), without affecting HR. Similar results were obtained from SB203580 (2 nmol), a cell-permeable inhibitor of p38MAPK [38] (Figure 5). Those pretreatments also significantly shortened the pro-life phase to $60 \mathrm{~min}$ by shifting the prevailing phase of the 180min observation period toward the pro-death phase (Figure 5). On the other hand, pretreatment with the negative control, SB202474 (2 nmol) was ineffective against the phasic cardiovascular responses in the aCSFcontrol group or Mev-experimental group.

\section{Discussion and conclusions}

Based on a clinically relevant experimental model [6], the present study provided novel demonstrations that activation of both JNK and p38MAPK in RVLM sustains central cardiovascular regulation during the progression towards brain stem death. We further showed that mechanistically, phosphorylation of MAP2K4 or MAP2K6 is upstream to activation of JNK or p38MAPK during the pro-life phase, with nuclear activation of transcription factors ATF-2 or c-Jun as the downstream signals (Figure 6).

The present study identified a novel pro-life role for MAP2K4/JNK/ATF-2 or c-Jun signaling cascade, rather than Elk-1, in RVLM during experimental brain stem death. JNK is a critical determinant for survival of cardiomyocytes from hypoxia-induced apoptosis [30,42]. Activation of JNK and its downstream transcription factor c-Jun, rather than ERK pathway, also plays a critical role in the survival and proliferation of pulmonary artery endothelial cells induced by epoxyeicosatrienoic acid [43]. Phosphorylation of JNK at Thr183 and Tyr185 by upstream MAP2Ks, MAP2K4 or MAP2K7, is important for the activation of JNK pathway $[44,45]$. Activation of JNK1/2 by MAP2K4 is responsible for cell survival in primary human umbilical vein endothelial cells mediated by vascular endothelial growth factor receptor-3 [46].

The present study also identified a novel a pro-life role for MAP2K6/p38MAPK/ATF-2 or c-Jun signaling cascade in RVLM during experimental brain stem death. The p38MAPK-dependent signaling cascade mediates critical cellular survival response to stress [47]. Upregulation of p38MAPK plays an important role in survival from cecal ligation and puncture-induced sepsis in mice [48], and inhibits apoptosis or proinflammatory response to lipopolysaccharide in microglial BV-2 cells [26] or in macrophages RAW 264.7 cells [48] or tumor necrosis factor alpha (TNF $\alpha)$ in murine fibrosarcoma L929 cells [49]. On the other hand, a decrease in the expression of phosphorylated p38MAPK is accompanied by cell death in TNF $\alpha$-treated L929 cells [49]. Constitutive expression of MKK6 (the alternative name for MAP2K6) phosphorylates p38 MAPK and enhances the survival of osteoclasts [50]. Activation of ATF-2 by p38MAPK prevents accumulation of reactive oxygen species and cell death in mouse embryo fibroblast [27].

We demonstrated previously the engagement of hypoxia-inducible factor $1 \alpha$ (HIF-1 $\alpha$ )/heme oxygenase $1 /$ heat shock protein 70 (HSP70) signaling pathway induced by hypoxia and tropomyocine receptor kinase $\mathrm{B}$ (Trk B)/Ras/Raf signaling pathways activated by brainderived neurotrophic factor (BDNF) in RVLM during the pro-life phase of experimental brain stem death. Of interest is that a potential role for JNK to serve as a survival factor by phosphorylation of a number of cellular molecules, including C-Jun, AP-1 or Bcl-2, is suggested for myocytes against hypoxia-reoxygenation injury [51]. Decreased JNK phosphorylation induced by inhibition of Ras or Raf mediates cell apoptosis [52]; and inhibition of Ras and p38MAPK reduces BDNF-induced survival of ganglion neurons [53]. Activation of the p38MAPK pathway is also an early response to hypoxia for cell survival because p38MAPK inhibition abolishes cell survival from hypoxia in rat neonatal cardiac myocytes [54] or LNCaP cells [55] and phosphorylation of p38MAPK induced by hypoxiapreconditioning mediates the protection of cardiomyocyte from ischemic injury [56]. It follows that JNK or p38MAPK may participate in the pro-life phase of experimental brain stem death as a consequence of hypoxia or BDNF activation in RVLM. Further studies are required to delineate these implied signaling cascades.

The transcription factor c-Jun is one of the most consistent markers for neuronal fate and is determined by a transcriptional network comprising c-Jun, ATF-2 and JNKs [57]. Overexpression of c-Jun in rat pheochromocytoma PC12 cells renders them to be more resistant to apoptosis induced by okadaic acid [58] or serumdeprivation [59]. High levels of c-Jun mRNA and proteins even function as a neuronal survival or neurite 


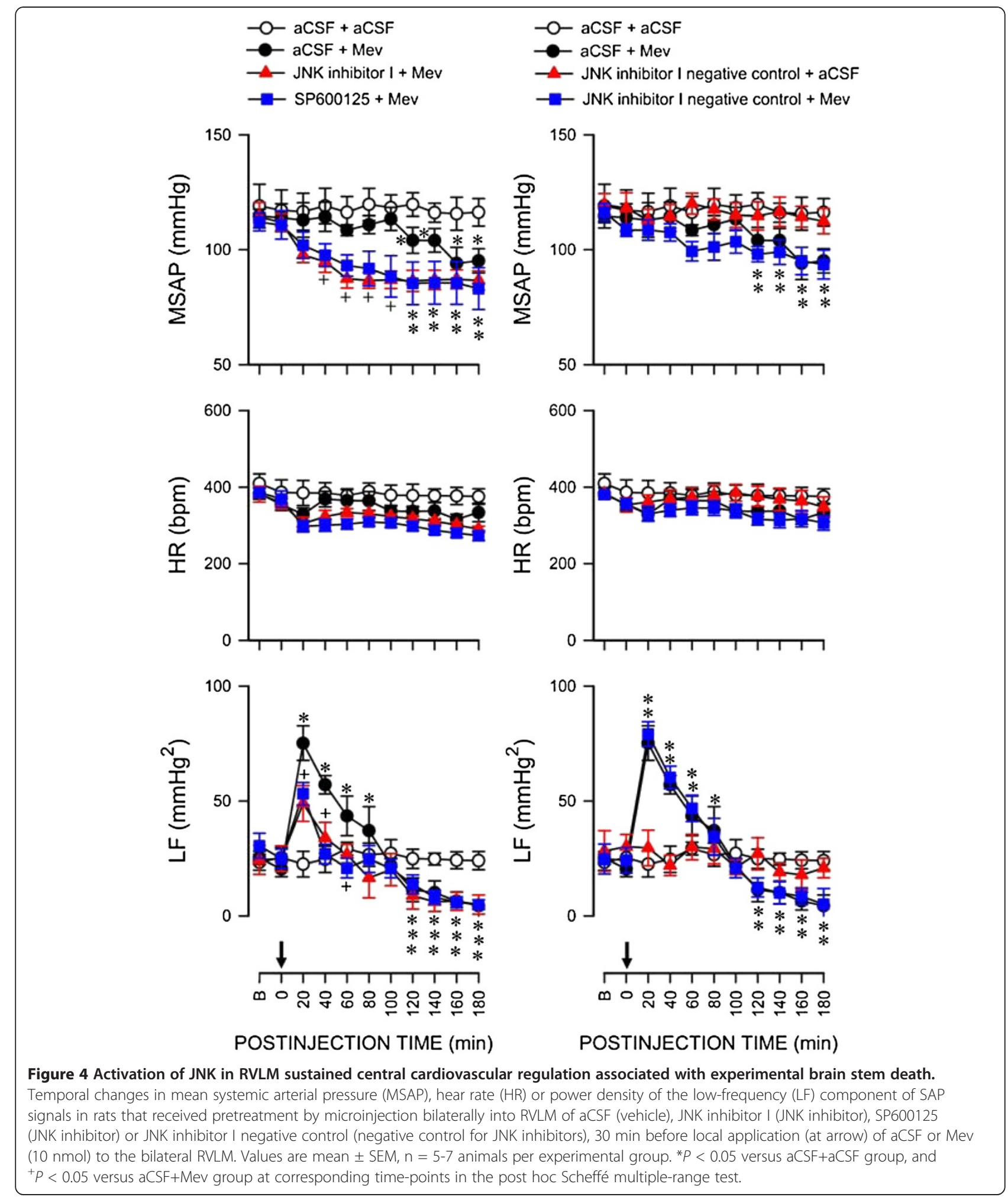

outgrowth signal for PC12 cell [58]. Mechanistically, it is most likely that ATF-2 or c-Jun in RVLM participates in the pro-life process by regulating its target proteins transcriptionally. Some of the known candidate proteins include HIF-1 $\alpha$ [60,61], HSP70 [62,63], anti-apoptotic Bcl-XL [64] and neuronal nitric oxide synthase [65]. In addition to transcriptional regulation, c-Jun also mediates posttranscriptional modification on HIF- $1 \alpha$ by 


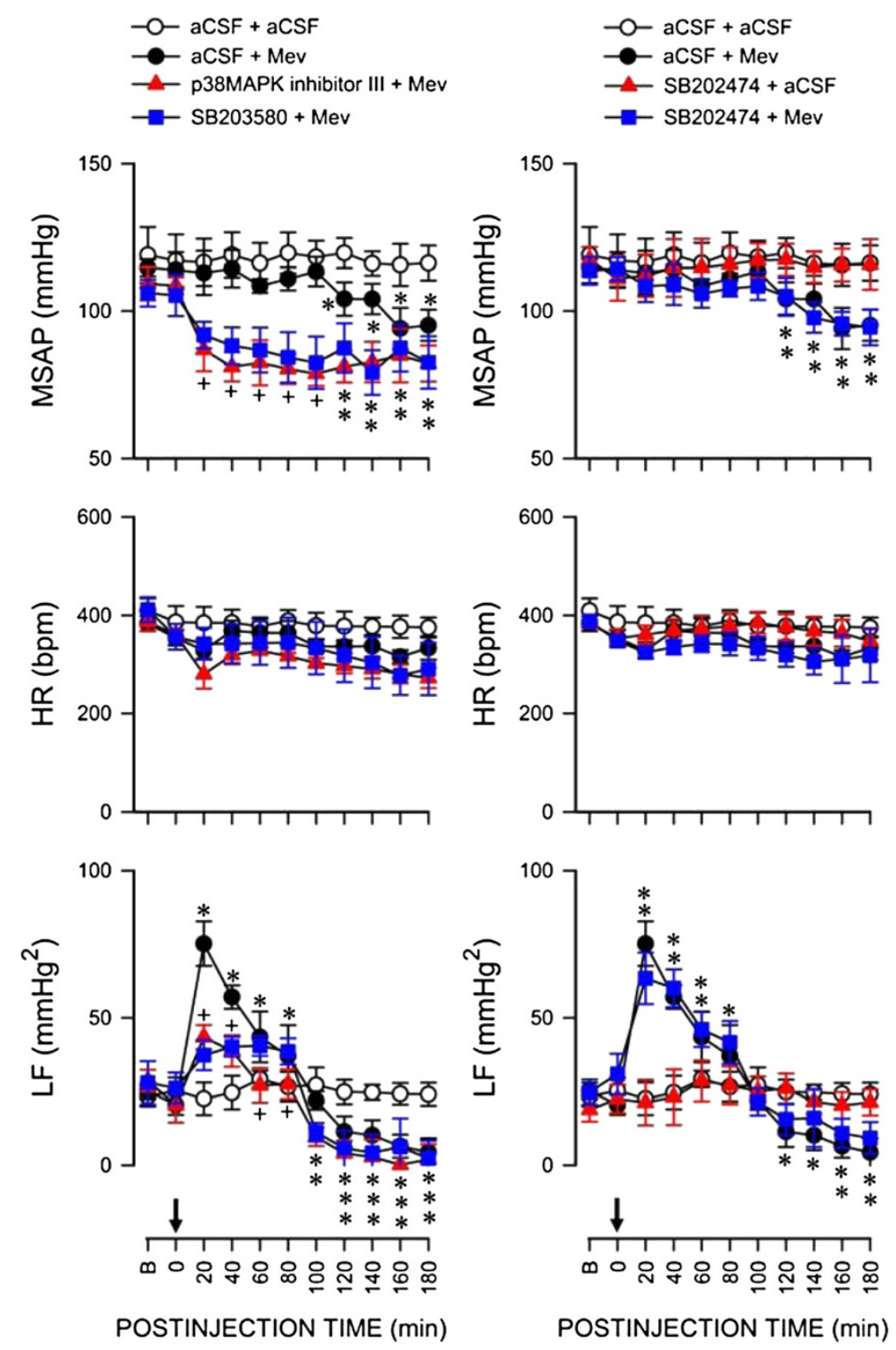

Figure 5 Activation of p38MAPK in RVLM sustained central cardiovascular regulation associated with experimental brain stem death. Temporal changes in MSAP, HR or power density of the LF component of SAP signals in rats that received pretreatment by microinjection bilaterally into RVLM of aCSF (vehicle), p38MAPK inhibitor III (p38MAPK inhibitor), SB203580 (p38MAPK inhibitor) or SB202474 (negative control for p38MAPK inhibitors), 30 min before local application (at arrow) of aCSF or Mev (10 nmol) to the bilateral RVLM. Values are mean \pm SEM, $n=5-7$ animals per experimental group. ${ }^{*} P<0.05$ versus aCSF+aCSF group, and ${ }^{+} P<0.05$ versus aCSF+Mev group at corresponding time-points in the post hoc Scheffé multiple-range test.

protecting it from proteasomal degradation [66]. Interestingly, all these proteins have been found to play a pro-life role in RVLM in our experimental model of brain stem death [16,67-71]. Fischer et al., [72] reported that marked increases in JNK and p38MAPK activity, coincident with an increase in phosphorylation of c-Jun and ATF-2, can be detected as early as $15-30$ min after rapid changes in hemodynamic load in Wistar rats. This time-course befits an active role for c-Jun and ATF-2 in
RVLM during the pro-life phase of experimental brain stem death.

In conclusion, the present study demonstrated that the MAP2K4/JNK or MAP2K6/p38MAPK signaling cascade in RVLM plays a pro-life role during experimental brain stem death by sustaining the central cardiovascular regulatory machinery via activating the transcription factors ATF-2 or c-Jun. This information provides further insights into the cellular mechanisms 


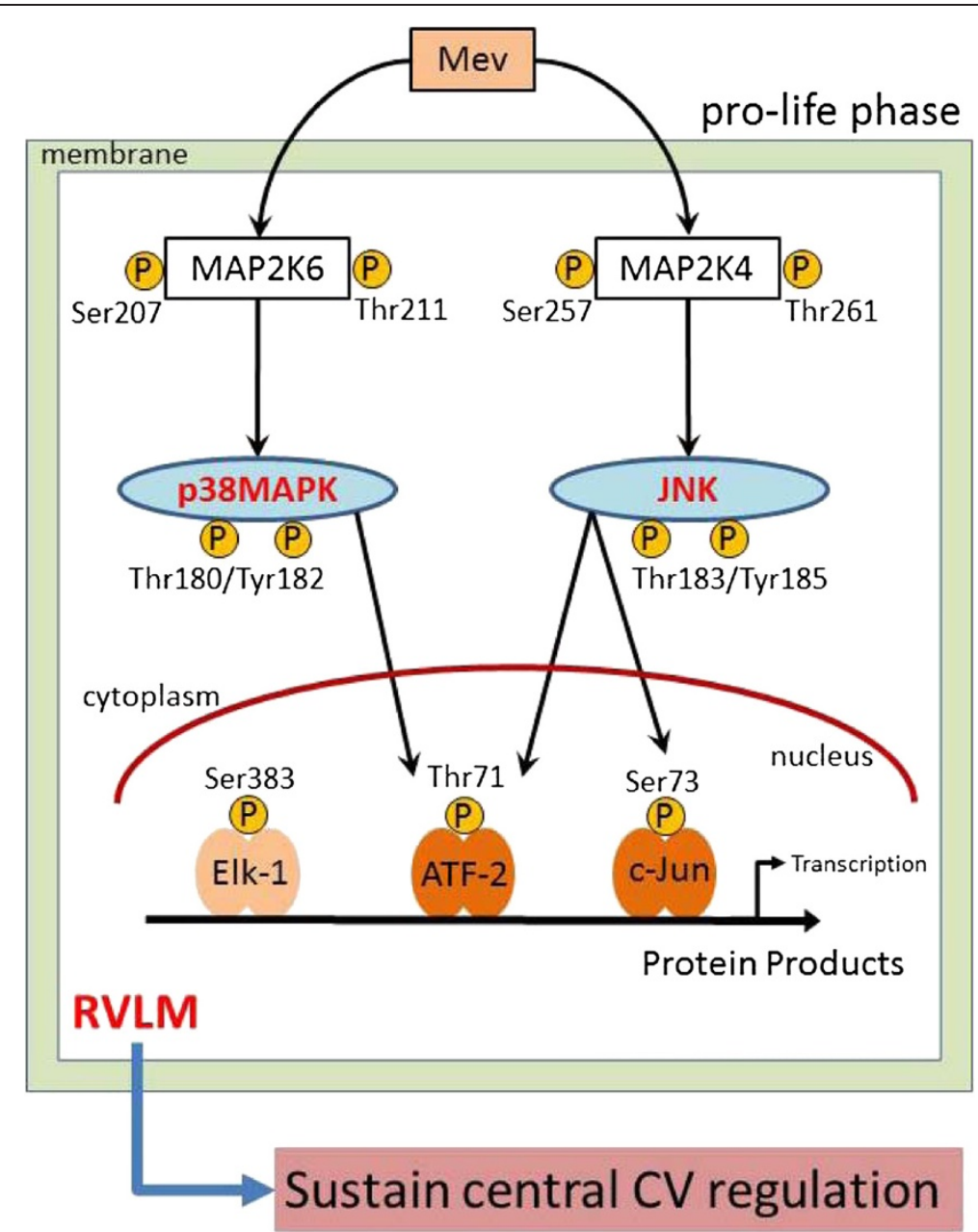

Figure 6 Schematic summary of the pro-life role of JNK and p38MAPK at RVLM during experimental brain stem death. Phosphorylation of MAP2K4 or MAP2K6, leading to activation of JNK or P38MAPK, and followed by nuclear activation of transcription factors ATF-2 or c-Jun, but not Elk-1, take place in RVLM preferentially during the pro-life phase of experimental brain stem death. Proteins that are downstream to these signaling cascades in turn sustain central cardiovascular regulation during the progression towards brain stem death. Abbreviation: ATF-2, activating transcriptional factor-2; JNK, c-Jun NH2-terminal kinase; CV, cardiovascular; MAPK, mitogen-activated protein kinase; MAP2K4, MAPK kinase 4; MAP2K6, MAPK kinase 6; Mev, mevinphos; p38MAPK, p38 mitogen-activated protein kinase; RVLM, rostral ventrolateral medulla.

of brain stem death, and offers new targets for the development of therapeutic interventions against this fatal phenomenon.

\section{Competing interests}

The author declares that she has no competing financial interests.

\section{Authors' contributions}

AYWC conceived the study, participated in experimental design, and drafted and revised the manuscript.

\section{Acknowledgements}

Supported by research grants NSC99-2628-B-182A-001-MY3, NSC99-2321-B182A-006 and NSC100-2321-B-182A-006 (AYWC) from the National Science Council, Taiwan, Republic of China.

Received: 17 September 2012 Accepted: 5 November 2012 Published: 17 November 2012

\section{References}

1. Anonymous: Report of the Medical Consultants on the Diagnosis of Death to the President's Commission for the Study of Ethical Problems in Medicine and Biomedical and Behavioral Research. J Am Med Assoc 1981, 246:2184-2186.

2. Anonymous: Diagnosis of brain death. Statement issued by the honorary secretary of the conference of medical royal colleges and their faculties in the United Kingdom on 11 October 1976. Br Med J 1976, 2:1187-1188.

3. Haupt WF, Rudolf J: European brain death codes: a comparison of national guidelines. J Neurol 1999, 246:432-437.

4. Hung TP, Chen ST: Prognosis of deeply comatose patients on ventilators. J Neurol Neurosurg Psychiatry 1995, 58:75-80.

5. Pallis C: ABC of Brain Stem Death. London: British Medical Journal Press; 1983.

6. Chan JYH, Chang AYW, Chan SHH: New insights on brain stem death: from bedside to bench. Prog Neurobiol 2005, 77:396-425.

7. Pearson G, Robinson F, Beers Gibson T, Xu BE, Karandikar M, Berman K, Cobb MH: Mitogen-activated protein (MAP) kinase pathways: regulation and physiological functions. Endocr Rev 2001, 22:153-183. 
8. Rose BA, Force T, Wang Y: Mitogen-activated protein kinase signaling in the heart: angels versus demons in a heart-breaking tale. Physiol Rev 2010, 90:1507-1546.

9. Yen DHT, Yen JC, Len WB, Wang LM, Lee CH, Chan SHH: Spectral changes in systemic arterial pressure signals during acute mevinphos intoxication in the rat. Shock 2001, 15:35-41.

10. Kuo TBJ, Yang CCH, Chan SHH: Selective activation of vasomotor component of SAP spectrum by nucleus reticularis ventrolateralis in rats. Am J Physiol 1997, 272:H485-H492.

11. Kuo TBJ, Yien HW, Hseu SS, Yang CCH, Lin YY, Lee LC, Chan SHH: Diminished vasomotor component of systemic arterial pressure signals and baroreflex in brain death. Am J Physiol 1997, 273:H1291-H1298.

12. Yien HW, Hseu SS, Lee LC, Kuo TBJ, Lee TY, Chan SHH: Spectral analysis of systemic arterial pressure and heart rate signals as a prognostic tool for the prediction of patient outcome in intensive care unit. Crit Care Med 1997, 25:258-266.

13. Yen DHT, Yien HW, Wang LM, Lee CH, Chan SHH: Spectral analysis of systemic arterial pressure and heart rate signals of patients with acute respiratory failure induced by severe organophosphate poisoning. Crit Care Med 2000, 28:2805-2811.

14. Chan JYH, Chan SHH, Chang AYW: Differential contributions of NOS isoforms in the rostral ventrolateral medulla to cardiovascular responses associated with mevinphos intoxication in the rat. Neuropharmacology 2004, 46:1184-1194.

15. Chan JYH, Chan SHH, Li FCH, Cheng HL, Chang AYW: Phasic cardiovascular responses to mevinphos are mediated through differential activation of cGMP/PKG cascade and peroxynitrite via nitric oxide generated in the rat rostral ventrolateral medulla by NOS I and II isoforms. Neuropharmacology 2005, 48:161-172.

16. Chan JYH, Cheng HL, Chou JLJ, Li FCH, Dai KY, Chan SHH, Chang AYW: Heat shock protein 60 or 70 activates NOS I- and inhibits NOS IIassociated signaling, and depresses mitochondrial apoptotic cascade during brain stem death. J Biol Chem 2007, 282:4585-4600.

17. Chan JYH, Wu CHY, Tsai CY, Cheng HL, Dai KY, Chan SHH, Chang AYW: Transcriptional up-regulation of nitric oxide synthase II by nuclear factor$\mathrm{KB}$ at rostral ventrolateral medulla in a rat mevinphos intoxication model of brain stem death. J Physiol 2007, 581:1293-1307.

18. Chan SHH, Sun EY, Chang AYW: Extracellular signal-regulated kinase 1/2 plays a pro-life role in experimental brain stem death via MAPK signalinteracting kinase at rostral ventrolateral medulla. J Biomed Sci 2010, 17:17-25.

19. Chan SHH, Chan JYH, Hsu KS, Li FCH, Sun EYH, Chen WL, Chang AYW: Amelioration of central cardiovascular regulatory dysfunction by tropomyocin receptor kinase $B$ in a mevinphos intoxication model of brain stem death. Br J Pharmacol 2011, 164:2015-2028.

20. Ruano D, Revilla E, Gavilan MP, Vizuete ML, Pintado C, Vitorica J, Castano A: Role of p38 and inducible nitric oxide synthase in the in vivo dopaminergic cells' degeneration induced by inflammatory processes after lipopolysaccharide injection. Neuroscience 2006, 140:1157-1168.

21. Adhikary G, Sun Y, Pearlman E: C-Jun $\mathrm{NH}_{2}$ terminal kinase (JNK) is an essential mediator of Toll-like receptor 2-induced corneal inflammation. J Leukoc Biol 2008, 83:991-997.

22. Harper SJ, LoGrasso P: Signalling for survival and death in neurones: the role of stress-activated kinases, JNK and p38. Cell Signal 2001, 13:299-310.

23. Zawada WM, Meintzer MK, Rao P, Marotti J, Wang X, Esplen JE, Clarkson $E D$, Freed CR, Heidenreich KA: Inhibitors of p38 MAP kinase increase the survival of transplanted dopamine neurons. Brain Res 2001, 891:185-196.

24. Rawal N, Parish C, Castelo-Branco G, Arenas E: Inhibition of JNK increases survival of transplanted dopamine neurons in Parkinsonian rats. Cell Death Differ 2007, 14:381-383.

25. Thornton TM, Rincon M: Non-classical p38 map kinase functions: cell cycle checkpoints and survival. Int J Biol Sci 2009, 5:44-51.

26. Svensson C, Part K, Künnis-Beres K, Kaldmäe M, Fernaeus SZ, Land T: Prosurvival effects of JNK and p38 MAPK pathways in LPS-induced activation of BV-2 cells. Biochem Biophys Res Commun 2011, 406:488-492.

27. Gutiérrez-Uzquiza Á, Arechederra M, Bragado P, Aguirre-Ghiso JA, Porras A: p38a mediates cell survival in response to oxidative stress via induction of antioxidant genes: effect on the p70S6K pathway. J Biol Chem 2012, 287:2632-2642.
28. Lamb JA, Ventura JJ, Hess P, Flavell RA, Davis RJ: JunD mediates survival signaling by the JNK signal transduction pathway. Mol Cell 2003, 11:1479-1489.

29. Chan SHH, Hsu KS, Huang CC, Wang LL, Ou CC, Chan JYH: NADPH oxidasederived superoxide anion mediates angiotensin Il-induced pressor effect via activation of p38 mitogen-activated protein kinase in the rostral ventrolateral medulla. Circ Res 2005, 97:772-780.

30. Shao Z, Bhattacharya K, Hsich E, Park L, Walters B, Germann U, Wang YM, Kyriakis J, Mohanlal R, Kuida K, Namchuk M, Salituro F, Yao YM, Hou WM, Chen X, Aronovitz M, Tsichlis PN, Bhattacharya S, Force T, Kilter H: c-Jun Nterminal kinases mediate reactivation of Akt and cardiomyocyte survival after hypoxic injury in vitro and in vivo. Circ Res 2006, 98:111-118.

31. Forrer $P$, Tamaskovic R, Jaussi R: Enzyme-linked immunosorbent assay for measurement of JNK, ERK, and p38 kinase activities. Biol Chem 1998, 379:1101-1111.

32. Yang CH, Shyr MH, Kuo TBJ, Tan PPC, Chan SHH: Effects of propofol on nociceptive response and power spectra of electroencephalographic and systemic arterial pressure signals in the rat: correlation with plasma concentration. J Pharmacol Exp Ther 1995, 275:1568-1574.

33. Li PL, Chao YM, Chan SHH, Chan JYH: Potentiation of baroreceptor reflex response by heat shock protein 70 in nucleus tractus solitarii confers cardiovascular protection during heatstroke. Circulation 2001, 103:2114-2119.

34. Ross CA, Ruggiero DA, Park DH, Joh TH, Sved AF, Fernandez-Pardal J, Saavedra JM, Reis DJ: Tonic vasomotor control by the rostral ventrolateral medulla: effect of electrical or chemical stimulation of the area containing $\mathrm{C} 1$ adrenaline neurons on arterial pressure, heart rate, and plasma catecholamines and vasopressin. J Neurosci 1984, 4:474-494.

35. Bonny C, Oberson A, Negri S, Sauser C, Schorderet DF: Cell-permeable peptide inhibitors of JNK: novel blockers of beta-cell death. Diabetes 2001, 50:77-82.

36. Bennett BL, Sasaki DT, Murray BW, O'Leary EC, Sakata ST, Xu W, Leisten JC, Motiwala A, Pierce S, Satoh Y, Bhagwat SS, Manning AM, Anderson DW: SP600125, an anthrapyrazolone inhibitor of Jun N-terminal kinase. Proc Natl Acad Sci U S A 2001, 98:13681-13686.

37. Laufer SA, Wagner GK, Kotschenreuther DA, Albrecht W: Novel substituted pyridinyl imidazoles as potent anticytokine agents with low activity against hepatic cytochrome P450 enzymes. J Med Chem 2003, 46:3230-3244

38. Lee JC, Laydon JT, McDonnell PC, Gallagher TF, Kumar S, Green D, McNulty D, Blumenthal MJ, Heys JR, Landvatter SW: A protein kinase involved in the regulation of inflammatory cytokine biosynthesis. Nature 1994, 372:739-746.

39. Ferrer I, Blanco R, Carmona M, Puig B, Domínguez I, Viñals F: Active, phosphorylation-dependent MAP kinases, MAPK/ERK, SAPK/JNK and p38, and specific transcription factor substrates are differentially expressed following systemic administration of kainic acid to the adult rat. Acta Neuropathol 2002, 103:391-407.

40. Sun HY, Wang NP, Halkos M, Kerendi F, Kin H, Guyton RA, Vinten-Johansen J, Zhao ZQ: Postconditioning attenuates cardiomyocyte apoptosis via inhibition of JNK and p38 mitogen-activated protein kinase signaling pathways. Apoptosis 2006, 11:1583-1593.

41. Chen KC, Chang LS: Notexin upregulates Fas and FasL protein expression of human neuroblastoma SK-N-SH cells through p38 MAPK/ATF-2 and JNK/c-Jun pathways. Toxicon 2010, 55:754-761.

42. Dougherty CJ, Kubasiak LA, Prentice H, Andreka P, Bishopric NH, Webster KA: Activation of c-Jun N-terminal kinase promotes survival of cardiac myocytes after oxidative stress. Biochem J 2002, 362:561-571.

43. Ma J, Zhang L, Han W, Shen T, Ma C, Liu Y, Nie X, Liu M, Ran Y, Zhu D: Activation of JNK/C-Jun is required for the proliferation, survival, and angiogenesis induced by EET in pulmonary artery endothelial cells. J Lipid Res 2012, 53:1093-1105.

44. Tournier C, Dong C, Turner TK, Jones SN, Flavell RA, Davis RJ: MKK7 is an essential component of the JNK signal transduction pathway activated by proinflammatory cytokines. Genes Dev 2001, 15:1419-1426.

45. Zou H, Li Q, Lin SC, Wu Z, Han J, Ye Z: Differential requirement of MKK4 and MKK7 in JNK activation by distinct scaffold proteins. FEBS Lett 2007, 581:196-202.

46. Salameh A, Galvagni F, Anselmi F, De Clemente C, Orlandini M, Oliviero S: Growth factor stimulation induces cell survival by c-Jun. ATF2dependent activation of BCl-XL. J Biol Chem 2010, 285:23096-23104. 
47. Shi GX, Jin L, Andres DA: A rit GTPase-p38 mitogen-activated protein kinase survival pathway confers resistance to cellular stress. Mol Cell Biol 2011, 31:1938-1948

48. Jang HJ, Kim YM, Tsoyi K, Park EJ, Lee YS, Kim HJ, Lee JH, Joe Y, Chung HT, Chang KC: Ethyl pyruvate induces heme oxygenase-1 through p38 mitogen-activated protein kinase activation by depletion of glutathione in RAW 264.7 cells and improves survival in septic animals. Antioxid Redox Signal 2012, 17:878-889.

49. Ye YC, Yu L, Wang HJ, Tashiro S, Onodera S, Ikejima T: TNFa-induced necroptosis and autophagy via supression of the p38-NF-KB survival pathway in L929 cells. J Pharmacol Sci 2011, 117:160-169.

50. Yamashita T, Kobayashi Y, Mizoguchi T, Yamaki M, Miura T, Tanaka S, Udagawa N, Takahashi N: MKK6-p38 MAPK signaling pathway enhances survival but not bone-resorbing activity of osteoclasts. Biochem Biophys Res Commun 2008, 365:52-257.

51. Shaw J, Kirshenbaum LA: Prime time for JNK-mediated Akt reactivation in hypoxia-reoxygenation. Circ Res 2006, 98:7-9.

52. Cheng Y, Qiu F, Ye YC, Tashiro S, Onodera S, Ikejima T: Oridonin induces G2/M arrest and apoptosis via activating ERK-p53 apoptotic pathway and inhibiting PTK-Ras-Raf-JNK survival pathway in murine fibrosarcoma L929 cells. Arch Biochem Biophys 2009, 490:70-75.

53. Mullen LM, Pak KK, Chavez E, Kondo K, Brand Y, Ryan AF: Ras/p38 and $\mathrm{PI} 3 \mathrm{~K} / \mathrm{Akt}$ but not Mek/Erk signaling mediate BDNF-induced neurite formation on neonatal cochlear spiral ganglion explants. Brain Res 2012, 1430:25-34.

54. Kacimi R, Chentoufi J, Honbo N, Long CS, Karliner JS: Hypoxia differentially regulates stress proteins in cultured cardiomyocytes: role of the p38 stress-activated kinase signaling cascade, and relation to cytoprotection. Cardiovasc Res 2000, 46:139-150.

55. Khandrika L, Lieberman R, Koul S, Kumar B, Maroni P, Chandhoke R, Meacham RB, Koul HK: Hypoxia-associated p38 mitogen-activated protein kinase-mediated androgen receptor activation and increased HIF-1alpha levels contribute to emergence of an aggressive phenotype in prostate cancer. Oncogene 2009, 28:1248-1260.

56. Wu X, Liu X, Zhu X, Tang C: Hypoxic preconditioning induces delayed cardioprotection through p38 MAPK-mediated calreticulin upregulation. Shock 2007, 27:572-577.

57. Herdegen T, Skene P, Bähr M: The c-Jun transcription factor-bipotential mediator of neuronal death, survival and regeneration. Trends Neurosci 1997, 20:227-231.

58. Dragunow M, Xu R, Walton M, Woodgate A, Lawlor P, MacGibbon GA, Young D, Gibbons H, Lipski J, Muravlev A, Pearson A, During M: c-Jun promotes neurite outgrowth and survival in PC12 cells. Brain Res Mol Brain Res 2000, 83:20-33.

59. Soeda S, Shinomiya K, Ochiai T, Koyanagi S, Toda A, Eyanagi R, Shimeno H: Plasminogen activator inhibitor-1 aids nerve growth factor-induced differentiation and survival of pheochromocytoma cells by activating both the extracellular signal-regulated kinase and c-Jun pathways. Neuroscience 2006, 141:101-108.

60. Alfranca A, Gutiérrez MD, Vara A, Aragonés J, Vidal F, Landázuri MO: c-Jun and hypoxia-inducible factor 1 functionally cooperate in hypoxiainduced gene transcription. Mol Cell Biol 2002, 22:12-22.

61. Choi $\mathrm{JH}$, Cho HK, Choi $\mathrm{YH}$, Cheong J: Activating transcription factor 2 increases transactivation and protein stability of hypoxia-inducible factor 1alpha in hepatocytes. Biochem J 2009, 424:285-296.

62. Kinouchi H, Sharp FR, Chan PH, Koistinaho J, Sagar SM, Yoshimoto T: Induction of c-fos, junB, c-jun, and hsp70 mRNA in cortex, thalamus, basal ganglia, and hippocampus following middle cerebral artery occlusion. J Cereb Blood Flow Metab 1994, 14:808-817.

63. Liu J, Zhang D, Mi X, Xia Q, Yu Y, Zuo Z, Guo W, Zhao X, Cao J, Yang Q, Zhu A, Yang W, Shi X, Li J, Huang C: p27 suppresses arsenite-induced Hsp27/ Hsp70 expression through inhibiting JNK2/c-Jun- and HSF-1-dependent pathways. J Biol Chem 2010, 285:26058-26065.

64. Salameh A, Galvagni F, Bardelli M, Bussolino F, Oliviero S: Direct recruitment of CRK and GRB2 to VEGFR-3 induces proliferation, migration, and survival of endothelial cells through the activation of ERK, AKT, and JNK pathways. Blood 2005, 106:3423-3431.

65. Wu W, Li Y, Schinco FP: Expression of c-jun and neuronal nitric oxide synthase in rat spinal motoneurons following axonal injury. Neurosci Lett 1994, 179:157-161.
66. Yu B, Miao ZH, Jiang Y, Li MH, Yang N, Li T, Ding J: c-Jun protects hypoxiainducible factor-1alpha from degradation via its oxygen-dependent degradation domain in a nontranscriptional manner. Cancer Res 2009, 69:7704-7712.

67. Chang C, Chang AYW, Chan SHH: Neuroprotective role of heat shock protein 70 in the rostral ventrolateral medulla during acute mevinphos intoxication in the rat. J Biomed Sci 2004, 11:748-755.

68. Li FCH, Chan $\mathrm{JYH}$, Chan $\mathrm{SHH}$, Chang AYW: In the rostral ventrolateral medulla, the 70-kDa heat shock protein (HSP70), but not HSP90, confers neuroprotection against fatal endotoxemia via augmentation of nitric-oxide synthase I (NOS I)/protein kinase G signaling pathway and inhibition of NOS II/peroxynitrite cascade. Mol Pharmacol 2005, 68:179-192.

69. Chang AYW, Chan JYH, Cheng HL, Tsai CY, Chan SHH: Hypoxia-inducible factor $1 /$ heme oxygenase 1 cascade as upstream signals in the prolife role of heat shock protein 70 at rostral ventrolateral medulla during experimental brain stem death. Shock 2009, 32:651-658.

70. Dai KY, Chan SHH, Chang AYW: Heme oxygenase-1 plays a pro-life role in experimental brain stem death via nitric oxide synthase 1/protein kinase G signaling at rostral ventrolateral medulla. J Biomed Sci 2010, 17:72-83.

71. Chan JYH, Tsai CY, Wu CH, Li FCH, Dai KY, Sun EYH, Chan SHH, Chang AYW: Sumoylation of hypoxia-inducible factor-1a ameliorates failure of brain stem cardiovascular regulation in experimental brain death. PLOS One 2011, 6:e17375.

72. Fischer TA, Ludwig S, Flory E, Gambaryan S, Singh K, Finn P, Pfeffer MA, Kelly RA, Pfeffer JM: Activation of cardiac c-Jun NH(2)-terminal kinases and p38-mitogen-activated protein kinases with abrupt changes in hemodynamic load. Hypertension 2001, 37:1222-1228.

doi:10.1186/1423-0127-19-96

Cite this article as: Chang: Pro-life role for c-Jun N-terminal kinase and p38 mitogen-activated protein kinase at rostral ventrolateral medulla in experimental brain stem death. Journal of Biomedical Science 2012 19:96.

\section{Submit your next manuscript to BioMed Central and take full advantage of:}

- Convenient online submission

- Thorough peer review

- No space constraints or color figure charges

- Immediate publication on acceptance

- Inclusion in PubMed, CAS, Scopus and Google Scholar

- Research which is freely available for redistribution 